Check for updates

Cite this: RSC Adv., 2019, 9, 26476

Received 9th July 2019

Accepted 14th August 2019

DOI: 10.1039/c9ra05246a

rsc.li/rsc-advances

\title{
First molecular electronic hyperpolarizability of series of $\pi$-conjugated oxazole dyes in solution: an experimental and theoretical study $\uparrow$
}

\author{
Luis M. G. Abegão, (D) *ab Ruben D. Fonseca, ${ }^{\text {cd }}$ Francisco A. Santos, ${ }^{a}$ José J. \\ Rodrigues, Jr, (D a Kenji Kamada, (D) e Cleber R. Mendonça, iD c Sandrine Piguel ${ }^{\text {fg }}$ \\ and Leonardo De Boni*c
}

In this work, we report the experimental and theoretical first molecular electronic hyperpolarizability ( $\left.\beta_{\mathrm{HRS}}\right)$ of eleven $\pi$-conjugated oxazoles compounds in toluene medium. The Hyper-Rayleigh Scattering (HRS) technique allowed the determination of the experimental dynamic $\beta_{\mathrm{HRS}}$ values, by exciting the compounds with a picosecond pulse trains from a Q-switched and mode-locked Nd:YAG laser tuned at $1064 \mathrm{~nm}$. Theoretical predictions based on time-dependent density functional theory level using the Gaussian 09 program package were performed with three different functionals (B3LYP, CAM-B3LYP, and M06-2X), to calculate both static and dynamic theoretical $\beta_{H R S}$ values. Good accordance was found between the experimental and theoretical values, in particular for the CAM-B3LYP and M06-2X functionals.

\section{Introduction}

Organic compounds with the ability to convert the frequency of incident light in its double, i.e., second harmonic generation (SHG), nowadays have vital importance in the optoelectronics field. ${ }^{1-3}$ The significance of that role has been highly competed for by both organic and inorganic compounds that possess nonlinear optical (NLO) responses, in particular, the first molecular hyperpolarizability, which is the NLO response studied in this work. ${ }^{\mathbf{4} 5}$ Such a response can be measured in a liquid medium by the hyper-Rayleigh scattering (HRS) ${ }^{6}$ technique. In general, inorganic compounds present a higher laser damage threshold than organic ones. On the other hand, the latter usually have a faster electronic response, making them more suitable, for example, for optical communications. ${ }^{7}$

${ }^{a}$ Departamento de Física, Universidade Federal de Sergipe, São Cristovão, SE, 49100-000, Brazil

${ }^{b}$ Department of Radiology and Bioimaging, School of Medicine, Yale University, 300 Cedar Street, New Haven, Connecticut 06520, USA

'Instituto de Física de São Carlos, Universidade de São Paulo, CP 369, 13560-970 São Carlos, SP, Brazil. E-mail: deboni@ifsc.usp.br

${ }^{d}$ Departamento de Fisica, Universidad Popular del Cesar, Barrio Sabana, Valledupar, Cesar, 2000004, Colombia

${ }^{e}$ National Institute of Advanced Industrial Science and Technology, Ikeda, Osaka 5638577, Japan

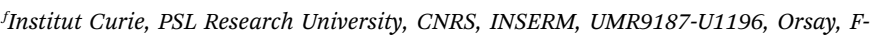
91405, France

${ }^{g}$ Université Paris Sud, Université Paris-Saclay, Orsay, F-91405, France

$\dagger$ Electronic supplementary information (ESI) available. See DOI: 10.1039/c9ra05246a
A $\pi$-conjugated system helps to promote the first electronic molecular hyperpolarizability, making dipolar organic compounds with a donor-bridge-acceptor (D-Br-A) configuration one of the most studied compounds in this field, ${ }^{8-10}$ although quadrupolar and octupolar configurations have also been explored..$^{11-13}$ Regardless of the multipole configuration shape, two fundamental aspects enhance the NLO response: the high degree of $\pi$-conjugation and the non-symmetric nature of the compounds. ${ }^{\mathbf{1 4 , 1 5}}$ The latter is an effect associated only to even-orders of NLO responses, while the first effect influences all NLO responses. ${ }^{16,17}$ Moreover, organic molecules tend to crystallize in the non-centrosymmetric form, which could amplify the SHG effect with the change of phase from liquid to solid, enhancing their potential as nonlinear optical switches. ${ }^{18,19}$

The series of $\pi$-conjugated molecular structures studied in this work belong to the oxazole family, in the particular case of oxazole dyes. This type of molecular structures, which possesses 2,5-diaryloxazole as its building block unit, has shown excellent transparency for wavelengths longer than $400 \mathrm{~nm},{ }^{20}$ making them extremely suitable to perform HRS measurements with a fixed excitation wavelength at the NIR region. Moreover, if the crystallization process of these type of compounds is successful, then their use as SHG organic crystals in the VIS-NIR region, which is the adequate region for optical communications, ${ }^{21-23}$ will be a real and concrete photonics application.

The fundamental principle of the HRS technique is to focus a laser beam with a certain frequency $\omega$ on a liquid sample and check whether the response of this light-matter interaction generates a light scattering with twice the frequency $(2 \omega)$ of the 
incident radiation, in order to determine the first molecular electronic hyperpolarizability $\left(\beta_{\mathrm{HRS}}\right)$ in an isotropic material. HRS is not the only available method to determine the $\beta_{\mathrm{HRS}}$ value. The traditional approach to achieve this quantity is known as the EFISH (Electric-Field Induced Second Harmonic Generation). ${ }^{24,25}$ There are some advantages of using HRS instead of EFISH, such as the non-requirement to identify the permanent dipole moment and the second molecular hyperpolarizability, nor to estimate the local fields, thus eliminating the need to apply a high static electric field in the studied sample.

In this work, we report for the first time experimental $\beta_{\mathrm{HRS}}$ values of eleven $\pi$-conjugated oxazole dyes in a solution of toluene, by using the HRS technique. Theoretical values of $\beta_{\mathrm{HRS}}$, calculated by using three different functionals (B3LYP, CAMB3LYP, and M06-2X) with basis sets 6-311G++(d,p) and 6$311 \mathrm{G}+(\mathrm{d}, \mathrm{p})$ are also reported, to best of our knowledge, for the first time, used to check accordance between experimental and theoretical data.

\section{Experimental}

The molecular structures of the eleven oxazoles dyes studied, are shown in Fig. 1 and are designated henceforth as $\mathbf{8 j}$ $\left(\mathrm{C}_{21} \mathrm{H}_{18} \mathrm{~N}_{2} \mathrm{O}_{4}\right), \quad 9 k \quad\left(\mathrm{C}_{17} \mathrm{H}_{16} \mathrm{~N}_{2} \mathrm{O}_{4}\right), \quad 10 b \quad\left(\mathrm{C}_{19} \mathrm{H}_{17} \mathrm{NO}_{3}\right), \quad$ 10d $\left(\mathrm{C}_{21} \mathrm{H}_{15} \mathrm{NO}\right), \quad$ 11b $\left(\mathrm{C}_{20} \mathrm{H}_{19} \mathrm{NO}_{4}\right), \quad$ 13k $\left(\mathrm{C}_{19} \mathrm{H}_{18} \mathrm{~N}_{2} \mathrm{O}_{4}\right), \quad$ 16b $\left(\mathrm{C}_{19} \mathrm{H}_{15} \mathrm{NO}_{3}\right), \quad 16 \mathrm{c} \quad\left(\mathrm{C}_{18} \mathrm{H}_{10} \mathrm{~N}_{2} \mathrm{O}\right), \quad 16 \mathrm{~d} \quad\left(\mathrm{C}_{21} \mathrm{H}_{13} \mathrm{NO}\right), \quad 16 \mathrm{e}$ $\left(\mathrm{C}_{18} \mathrm{H}_{10} \mathrm{~F}_{3} \mathrm{NO}\right)$ and $16 \mathrm{f}\left(\mathrm{C}_{16} \mathrm{H}_{10} \mathrm{~N}_{2} \mathrm{O}\right)$. The information about the synthesis and preparation of these compounds can be found elsewhere. ${ }^{20}$ Each compound was diluted in five different

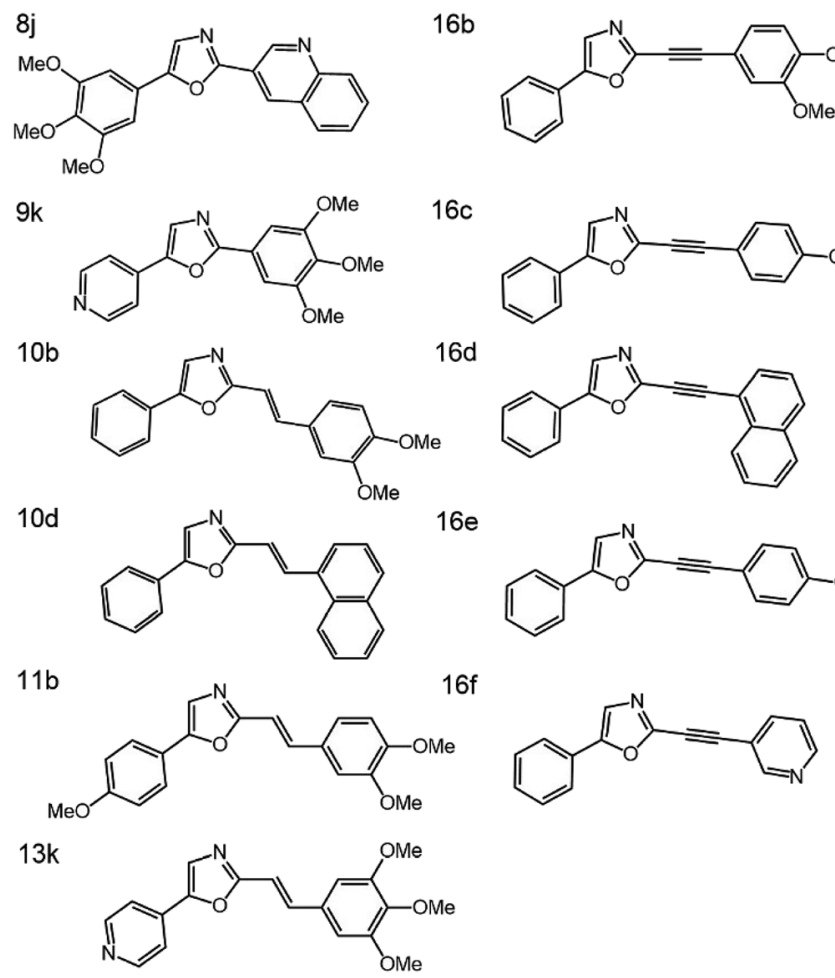

Fig. 1 Molecular structures of the studied samples. concentrations, ranging from $1 \times 10^{17}$ to $3 \times 10^{18}$ molecules per $\mathrm{cm}^{3}$, in toluene, and kept in a fused quartz cuvette with $10 \mathrm{~mm}$ long optical path. Toluene was used as a solvent due to its excellent solubility presented at the higher samples concentrations also, because it is from the family of hydrocarbon solvents belonging, which usually are inert to reagents of all kinds, besides being neither acid nor basic, under the Lewis or Brønsted perspective.

The classical 90 degrees optical geometry was used in the HRS experimental setup (Fig. 2), which has the same optical configuration presented by Clays and Persoons, ${ }^{6}$ with some variations introduced by Franzen $e t$ al. ${ }^{26}$ to take advantage of the experimental optoelectronic setup described in the next paragraph.

The excitation light source used was Q-switched and modelocked solid-state laser (Nd:YAG), with a variable repetition rate from $3 \mathrm{~Hz}$ to $800 \mathrm{~Hz}$, which produces a train with about 30 laser pulses separated from each other of approximately $13 \mathrm{~ns}$. Each pulse of the pulse train has $100 \mathrm{ps}$ of pulse width. The onset, depicted in Fig. 2a, shows the intrinsic intensity distribution of the pulse train at $1064 \mathrm{~nm}$, corresponding to the laser's fundamental frequency $(\omega)$.

The laboratory and molecular coordinates system of references were defined as $(X, Y, Z)$ and $(x, y, z)$, respectively, as illustrated in Fig. $2 \mathrm{~b}$. The propagation of the laser beam is along the $X$-axis, defined as the laboratory system of reference, in which the first element is a computer-controlled shutter (S), set up closed during the dark count rate (DCR) and opened only during the measurements of the sample, i.e., when acquiring the HRS signal. The second element consists of a set of cross polarizers $(\mathrm{P})$ to control two optical parameters: the maximum average power and to fix the linear polarization of the laser beam, which is always in the $Z$-axis. The third element is a beam splitter (BS), used to deviate a small portion of the laser beam into a fast-amplified photodetector (APD), which has a rise time around one nanosecond. The APD acquired signal is used as the

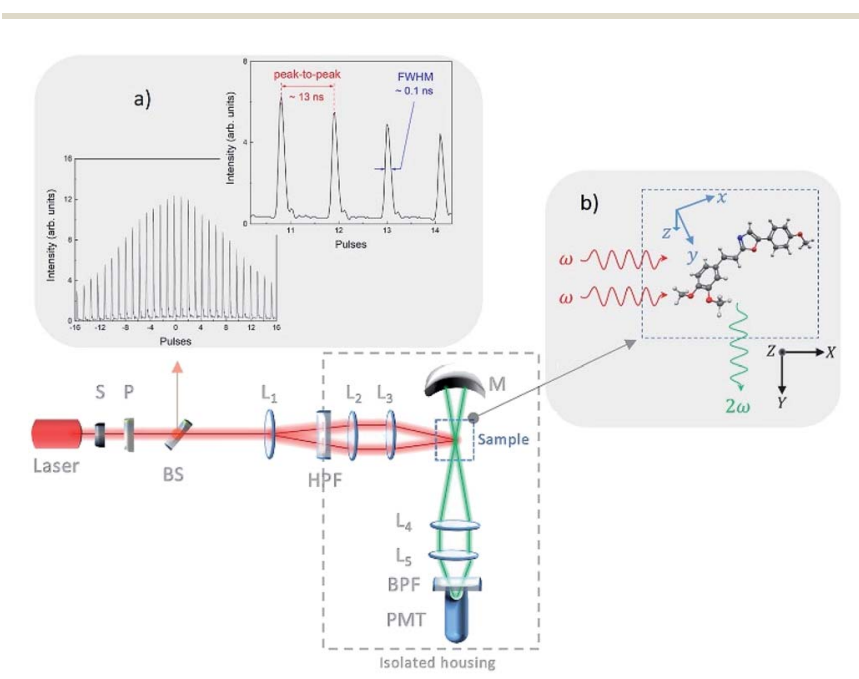

Fig. 2 HRS experimental setup, with the (a) intrinsic intensity distribution of the pulse train at $1064 \mathrm{~nm}$; and (b) the laboratory and molecular coordinates system of references. 
reference pulse train intensities, as shown on Fig. 2a. After the $\mathrm{BS}$, the laser beam is first expanded then collimated and finally focused on the sample by L1, L2, and L3 lenses respectively. This procedure avoids damaging the cuvette wall. The scattered signal $(2 \omega)$, i.e., the HRS signal, is collected perpendicularly ( $Y$ axis) to the excitation beam by a photomultiplier tube (PMT). A bandpass filter with $10 \mathrm{~nm}$ band at $532 \mathrm{~nm}$ was placed in front of the PMT window, to minimize any possible scattered excitation light. Also, a spherical mirror (M) is used to improve the PMT signal-to-noise ratio. More details about the experimental setup can be found elsewhere. ${ }^{11,27,28}$

HRS experiment is performed by measuring the intensity of the incoherently scattered frequency-doubled light, generated by the interaction of an intense laser beam with an isotropic solution. If the solution presents negligible molar absorptivity at harmonic wavelength, the relation between scattered intensity at double-frequency $\left(I_{2 \omega}\right)$ and intensity of the excitation beam $\left(I_{\omega}\right)$ is given by eqn $(1) .{ }^{29} G$ is a parameter that includes experimental factors such as the scattering geometry, local field factors at $\omega$ and $2 \omega$, and photon collection efficiency. $N$ represents the molar concentration of the used samples and $\left\langle\beta_{\mathrm{HRS}}\right\rangle$ is the orientational average first molecular electronic hyperpolarizability squared. The subscripts $\mathrm{s}$ and $\mathrm{c}$ refer to the solvent and chromophore respectively.

$$
I_{2 \omega}=G\left(N_{\mathrm{s}}\left\langle\beta_{\mathrm{HRS}}{ }^{2}\right\rangle_{\mathrm{s}}+N_{\mathrm{c}}\left\langle\beta_{\mathrm{HRS}}{ }^{2}\right\rangle_{\mathrm{c}}\right) I_{\omega}^{2}
$$

By considering a plane-polarized incident light beam, while the observation of the scattered signal on a frequency doubled light is made perpendicular to the propagation plane, the full HRS intensity could be described by eqn $2 .\left\langle\beta_{Z Z Z}{ }^{2}\right\rangle$ and $\left\langle\beta_{Z X X}{ }^{2}\right\rangle$ correspond to the orientationally averaged tensor components, here we adopted the laboratory coordinate system of reference.

$$
\left\langle\beta_{\mathrm{HRS}}\right\rangle=\sqrt{\left\langle\beta_{Z Z Z}{ }^{2}\right\rangle+\left\langle\beta_{Z X X}{ }^{2}\right\rangle}
$$

Eqn (3a) and (3b) show the relationship between those molecular averages and the molecular first-order hyperpolarizability tensor components, given by the molecular system of reference $(x, y, z)$, without assuming Kleinman's conditions of symmetry. ${ }^{30-32}$

$$
\begin{aligned}
\left\langle\beta_{Z Z Z}{ }^{2}\right\rangle= & \frac{1}{7} \sum_{\zeta}^{x, y, z} \beta_{\zeta \zeta \zeta}^{2}+\frac{4}{35} \sum_{\zeta \neq \eta}^{x, y, z} \beta_{\zeta \zeta \eta}^{2}+\frac{2}{35} \sum_{\zeta \neq \eta}^{x, y, z} \beta_{\zeta \zeta \zeta} \beta_{\zeta \eta \eta} \\
& +\frac{4}{35} \sum_{\zeta \neq \eta}^{x, y, z} \beta_{\eta \zeta \zeta} \beta_{\zeta \zeta \eta}+\frac{4}{35} \sum_{\zeta \neq \eta}^{x, y, z} \beta_{\zeta \zeta \zeta} \beta_{\eta \eta \zeta}+\frac{1}{35} \sum_{\zeta \neq \eta}^{x, y, z} \beta_{\eta \zeta \zeta}{ }^{2} \\
& +\frac{4}{105} \sum_{\zeta \neq \eta \neq \xi}^{x, y, z} \beta_{\zeta \zeta \eta} \beta_{\eta \xi \xi}+\frac{1}{105} \sum_{\zeta \neq \eta \neq \xi}^{x, y, z} \beta_{\eta \zeta \zeta} \beta_{\eta \xi \xi} \\
& +\frac{4}{105} \sum_{\zeta \neq \eta \neq \xi}^{x, y, z} \beta_{\zeta \zeta \eta} \beta_{\xi \xi \eta}+\frac{2}{210} \sum_{\zeta \neq \eta \neq \xi}^{x, y, z} \beta_{\zeta \eta \xi}{ }^{2} \\
& +\frac{4}{105} \sum_{\zeta \neq \eta \neq \xi}^{x, y, z} \beta_{\zeta \eta \xi} \beta_{\eta \zeta \xi}
\end{aligned}
$$

$$
\begin{aligned}
\left\langle\beta_{Z X X}{ }^{2}\right\rangle= & \frac{1}{35} \sum_{\zeta}^{x, y, z} \beta_{\zeta \zeta \zeta}{ }^{2}+\frac{4}{105} \sum_{\zeta \neq \eta}^{x, y, z} \beta_{\zeta \zeta \zeta} \beta_{\zeta \eta \eta}-\frac{2}{35} \sum_{\zeta \neq \eta}^{x, y, z} \beta_{\zeta \zeta \zeta} \beta_{\eta \eta \zeta} \\
& +\frac{8}{105} \sum_{\zeta \neq \eta}^{x, y, z} \beta_{\zeta \zeta \eta}{ }^{2}+\frac{3}{35} \sum_{\zeta \neq \eta}^{x, y, z} \beta_{\zeta \eta \eta}{ }^{2}-\frac{2}{35} \sum_{\zeta \neq \eta}^{x, y, z} \beta_{\zeta \zeta \eta} \beta_{\eta \zeta \zeta} \\
& +\frac{1}{35} \sum_{\zeta \neq \eta \neq \xi}^{x, y, z} \beta_{\zeta \eta \eta} \beta_{\zeta \xi \xi}-\frac{2}{105} \sum_{\zeta \neq \eta \neq \xi}^{x, y, z} \beta_{\zeta \zeta \xi} \beta_{\eta \eta \xi} \\
& -\frac{2}{105} \sum_{\zeta \neq \eta \neq \xi}^{x, y, z} \beta_{\zeta \zeta \eta} \beta_{\eta \xi \xi}+\frac{2}{35} \sum_{\zeta \neq \eta \neq \xi}^{x, y, z} \beta_{\zeta \eta \xi}{ }^{2} \\
& -\frac{2}{105} \sum_{\zeta \neq \eta \neq \xi}^{x, y, z} \beta_{\zeta \eta \xi} \beta_{\eta \zeta \xi}
\end{aligned}
$$

\section{Theoretical details}

Quantum-chemical calculations (QCC) were performed at the density functional theory ${ }^{33}$ by using the well-known exchangecorrelation functional Becke, three-parameter, Lee-Yang-Parr (B3LYP), ${ }^{34}$ as well its long-range corrected version which includes the Coulomb-attenuating method (CAM-B3LYP). ${ }^{35}$ Also, a variation of a hybrid functional by Truhlar and Zhao (M06-2X), ${ }^{36}$ which is one of the suitable functionals to study non-covalent interactions, was used. A split-valence basis set with one additional polarization function for non-hydrogen atoms, one additional polarization function on hydrogen atoms, and diffuse functions on non-hydrogen atoms were used associated with families of methods described previously. Geometry optimization calculations were performed in toluene medium employing a polarizable continuum model (PCM), using the integral equation formalism variant (IEF-PCM), ${ }^{37}$ to obtain the geometrical structure input used for the simulation of the static $(\omega=0$ a.u.) and dynamic $(\omega=0.04282$ a.u.) components of the first-order hyperpolarizability tensor $\left(\beta_{\mathrm{ijk}}\right)$ in solvent medium. Gaussian 09 program package ${ }^{38}$ was used to perform all the calculations mentioned above. The optimized geometries are shown in ESI (Tables S1 to S6 $\dagger$ ).

When studying systems with a low-symmetry molecular structure, the hyperpolarizability tensor could be estimated more accurately by using the rotational invariance concept, ${ }^{39}$ combined with the use of the mixed spherical-Cartesian formalism. ${ }^{40}$ Eqn (4) expresses, under this formalism, the orientational averaged first-order hyperpolarizability squared $\left\langle\beta_{\mathrm{HRS}}\right\rangle$, in which the molecular $\beta_{\mathrm{HRS}}$ tensor (symmetric 3rd rank tensor) is decomposed as the sum of a dipolar $(J=1)$ and an octupolar $(J=3)$ tensorial form. The relationships between the dipolar and octupolar components and the Cartesian components of $\beta$ are described in eqn (5). The combination between eqn (4) and (5) allows the final theoretical values estimation regarding the static and dynamic first-order hyperpolarizabilities.

For the study of systems with very low-symmetry, it is convenient to explore the concept of rotational invariance to represent of the hyperpolarizability tensor and to use the mixed 
spherical-Cartesian formalism to discuss HRS measurements in terms of multipolar components. ${ }^{39}$ In this formalism, ${ }^{40}$ the orientational averaged first-order hyperpolarizability squared $\left\langle\beta_{\mathrm{HRS}}\right\rangle$ is expressed by eqn (4), in which the molecular $\beta_{\mathrm{HRS}}$ tensor (symmetric $3 \mathrm{rd}$ rank tensor) is decomposed as the sum of a dipolar $(J=1)$ and an octupolar $(J=3)$ tensorial form. The relationships between the dipolar and octupolar components and the Cartesian components of $\beta$ are described in eqn (5). The combination between eqn (4), 5(a) and 5(b) allows estimate the final theoretical values for the static and dynamic first-order hyperpolarizabilities.

$$
\begin{gathered}
\left\langle\beta_{\mathrm{HRS}}\right\rangle=\sqrt{\frac{2}{9}\left|\beta_{J=1}\right|^{2}+\frac{2}{21}\left|\beta_{J=3}\right|^{2}} \\
\left|\beta_{J=1}\right|^{2}=\frac{3}{5} \sum_{\zeta}^{x, y, z} \beta_{\zeta \zeta \zeta}{ }^{2}+\frac{6}{5} \sum_{\zeta \neq \eta}^{x, y, z} \beta_{\zeta \zeta \zeta} \beta_{\zeta \eta \eta}+\frac{3}{5} \sum_{\zeta \neq \eta}^{x, y, z} \beta_{\eta \zeta \zeta}{ }^{2} \\
+\frac{3}{5} \sum_{\zeta \neq \eta \neq \xi}^{x, y, z} \beta_{\zeta \eta \eta} \beta_{\zeta \xi \xi} \\
\left|\beta_{J=3}\right|^{2}=\frac{2}{5} \sum_{\zeta}^{x, y, z} \beta_{\zeta \zeta \zeta^{2}}-\frac{6}{5} \sum_{\zeta \neq \eta}^{x, y, z} \beta_{\zeta \zeta \zeta} \beta_{\zeta \eta \eta}+\frac{12}{5} \sum_{\zeta \neq \eta}^{x, y, z} \beta_{\eta \zeta \zeta}^{2} \\
-\frac{3}{5} \sum_{\zeta \neq \eta \neq \xi}^{x, y, z} \beta_{\zeta \eta \eta} \beta_{\zeta \xi \xi}+\sum_{\zeta \neq \eta \neq \xi}^{x, y, z} \beta_{\zeta \eta \xi}{ }^{2}
\end{gathered}
$$

\section{Results and discussion}

The absorbance spectra presented in the ESI (Fig. S1 to S11 $\dagger$ ) of all the studied samples in toluene medium showed that there is not any absorption at $532 \mathrm{~nm}$. Therefore, an induced twophoton absorption process is also neglected when exciting the samples with $1064 \mathrm{~nm}$. In fact, the absorbance spectra show that all samples present a transparency window that starts at approximately $425 \mathrm{~nm}$, going to NIR region, indicating no absorption of the HRS signal by the samples. Moreover, the twophoton absorption spectra of some compounds have already been reported elsewhere, ${ }^{41}$ revealing that for excitation wavelengths longer than $900 \mathrm{~nm}$ the two-photon absorption crosssection is null. The probability of a three-photon excitation, which is a fifth-order NLO process, has significantly lower efficiency than its homolog third-order NLO phenomenon; thus, the fluorescence emission of the samples triggered by multiphoton excitation is completely ruled out. The optical signal acquired on the PMT, under this experimental setup, is considered a SHG effect at $532 \mathrm{~nm}$, which is confirmed by the quadratic dependence of the HRS signal $(I(2 \omega))$ as a function of the excitation intensity $(I(\omega))$, as can be observed in Fig. 3 for compound $\mathbf{8 j}$.

The data analysis of the compound $\mathbf{8 j}$ dissolved in toluene is shown in Fig. 3, as an example of how to interpret a typical experimental HRS result. The open circles are the experimental points acquired at 90 degrees by the PMT when the sample is shined by the intense pulsed laser at $1064 \mathrm{~nm}$. The five solid lines represent the second order polynomial best fits for five

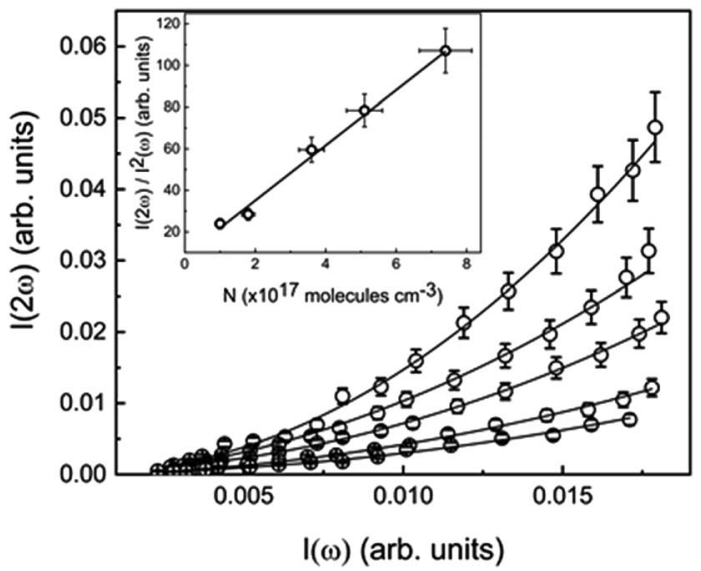

Fig. 3 Experimental first hyperpolarizability scattering signals for $8 \mathrm{j}$ dissolved in toluene (open circles). It shows the quadratic dependence of the HRS as a function of the pump intensity (1064 nm). Solid lines are the second order polynomial best fits for each concentration. The insets show the linear dependence between the hyperpolarizability signal $\left(/(2 \omega) / I^{2}(\omega)\right)$ and the sample concentrations with the respective best linear fit (solid line).

different concentrations, which are in accordance with the fundamental HRS physical principle described in the literature. ${ }^{6}$ It is also possible to observe, in the inset of Fig. 3, the linear behaviour between the intensity of the first-order hyperpolarizability signal $(I(2 \omega) / I 2(\omega))$ and the sample concentration, allowing the comparison with the external reference compound, that, in this particular study, was the para-nitroaniline (PNA). The evaluation of the dynamic first-order hyperpolarizability, for this respective sample (8j), was made by calculating the ratio between the angular coefficients $(\alpha)$ of the sample with the one obtained from the reference molecule (PNA), by using eqn (6). The complete set of data analysis for all compounds is presented in the ESI (Fig. S12-S21†).

$$
\beta_{\mathrm{HRS} \text { (sample) }}=\beta_{\mathrm{HRS} \text { (reference) }} \sqrt{\frac{\alpha_{\text {sample }}}{\alpha_{\text {reference }}}}
$$

As mentioned before, the chosen compound to use as an external reference was PNA, with a $\beta_{2 \omega}$ value of $18 \times 10^{-30} \mathrm{~cm}^{4}$ statvolt $^{-1}$ in toluene medium..$^{42}$ The highest HRS signal recorded corresponds to the compound 16c which is three times higher than the reference compound, while the lowest signal belongs to compound $\mathbf{1 0 c}$, which has approximately the same value of the reference compound. All the theoretical results obtained by using the functionals B3LYP, M06-2X and CAMB3LYP, with the basis sets $6-311+\mathrm{G}(\mathrm{d}, \mathrm{p})$ and $6-311++\mathrm{G}(\mathrm{d}, \mathrm{p})$, as well the experimental ones are presented in the ESI (Tables S7 and $\mathrm{S} 8 \dagger$ ).

Fig. 4(a) and (b) compare the theoretical results with the previous mentioned functionals by using a basis set with diffuse functions only on heavy atoms (non-H atoms), while Fig. 4(c) and (d) show the comparison by using the same functionals, but now applying a basis set with both diffuse functions on $\mathrm{H}$ and non-H atoms. The theoretical results were considerably 

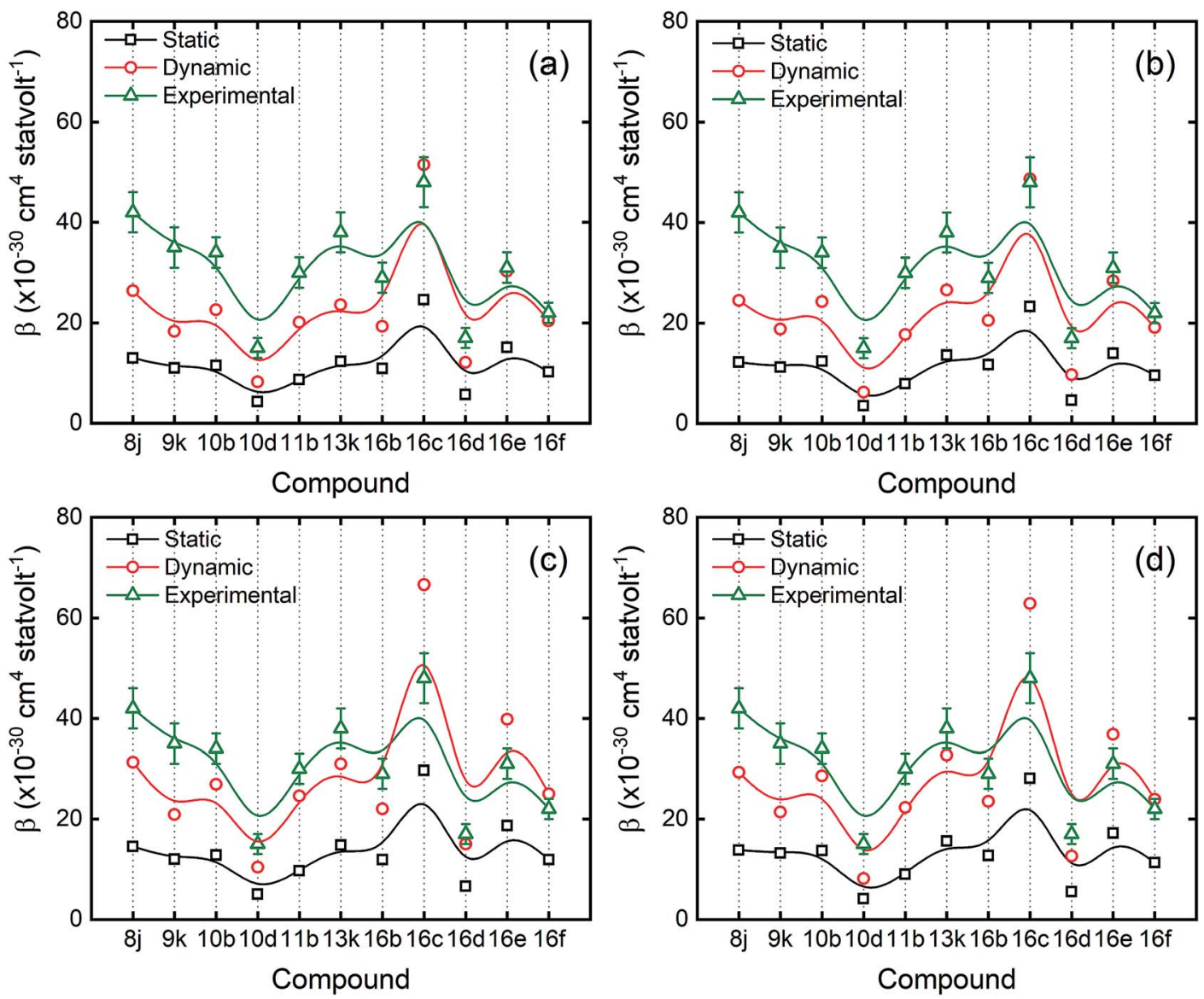

Fig. 4 Values for theoretical static $\left(\beta_{0}\right)$ (squares), theoretical dynamic ( $\beta_{\text {HRS }}$ ) (circles) and experimental (triangles) first hyperpolarizability $(\beta)$ (in $10^{-30} \mathrm{~cm}^{4}$ statvolt ${ }^{-1}$ ) in toluene medium of the investigated compounds; (a) represents the comparison between experimental and $\mathrm{M06}-2 \mathrm{X}$ method and (b) with CAM-B3LYP, with the basis set 6-311+G(d,p); while (c) and (d) represents the comparison between experimental and M06$2 X$, and CAM-B3LYP, respectively but by using the basis set $6-311++G(d, p)$. The solid lines are just guidelines to the eye.

underestimated for every compound when compared to experimental ones, calculated with the B3LYP (data shown in Tables S7 and S8†). However, when calculated with M06-2X and CAMB3LYP, the results are less underestimated when compared to experimental ones, in particular when using with the basis set which includes both diffuse functions on $\mathrm{H}$ and non- $\mathrm{H}$ atoms $(6-311++\mathrm{G}(\mathrm{d}, \mathrm{p}))$.

As one can see in Fig. 4, the tendency is very similar between experimental and theoretical values, for both plotted methods as a function of the molecules. In other words, when the experimental value increases for some molecule, the theoretical one follows the same tendency. In fact, by applying the basis set $6-311++G(d, p)$, each theoretical value shows a small increment on their value when compared to 6-311+G(d,p). However, it still below to the experimental ones, except for compounds 16c and 16e. This behaviour suggests that by using $6-311++G(d, p)$ basis set, which takes into account diffuse functions on both non- $\mathrm{H}$ and $\mathrm{H}$ atoms, helps to improve the simulation on molecular hyperpolarizabilities. The contrast of performance between the functionals B3LYP and CAM-B3LYP is entirely accepted since CAM-B3LYP is the B3LYP corrected version taking into account the Coulomb-attenuating method.
When comparing the values of the experimental first hyperpolarizability with the theoretical values of the electric dipole moment, a similar trend to the one depicted in Fig. 4 is obtained, as one can see in the ESI (Fig. S22†).

\section{Conclusions}

In summary, we reported the first-order hyperpolarizability of a new class of chromophores based on the oxazole scaffold substituted with styryl or alkynyl groups. All compounds showed values for the first molecular hyperpolarizability higher than the standard sample. As mentioned elsewhere, ${ }^{43-45}$ centrosymmetrical molecular structures should have an extremely low second-order nonlinear optical signal. Consequently, it was expected that the compounds investigated in this work, which have a low centrosymmetric structure nature, present significant HRS signal, such as the compound $\mathbf{1 6 c}$ which presented a value three times higher than the reference compound.

The samples were pumped with a pulsed laser at $1064 \mathrm{~nm}$, which is a wavelength far away from the absorption region. In this condition, we excluded any contribution from the one- and two-photon absorption process to populate any excited states. 
Therefore, all $\beta_{\mathrm{HRS}}$ experimental values presented in this work are due to pure electronic effects from the SHG in solution, suggesting that these compounds can be used as raw materials for developing nonlinear second-order photonics devices.

The theoretical values obtained by the QCC performed at the density functional theory by using the Gaussian 09 program package showed reasonable accordance with the experimental ones. In particular, when the calculations were performed with the functionals CAM-B3LYP and M06-2X.

\section{Conflicts of interest}

There are no conflicts to declare.

\section{Acknowledgements}

The authors acknowledge the financial support from Coordenação de Aperfeiçoamento de Pessoal de Nível Superior Brazil (CAPES) - Finance Code 001, CNPq/MCT, FAPITEC, Nanofoton Network, INCT-FOTÔNICA, FAPESP 2016/20886-1 and 2018/11283-7, Army Research Laboratory W911NF-17-10123 and Air Force Office of Scientific Research (FA9550-12-10028).

\section{References}

1 F. Castet, V. Rodriguez, J.-L. Pozzo, L. Ducasse, A. Plaquet and B. Champagne, Acc. Chem. Res., 2013, 46, 2656-2665.

2 H. Zhang, Q. Liao, X. Wang, Z. Xu and H. Fu, Nanoscale, 2015, 7, 10186-10192.

3 X.-S. Xing, R.-J. Sa, P.-X. Li, N.-N. Zhang, Z.-Y. Zhou, B.-W. Liu, J. Liu, M.-S. Wang and G.-C. Guo, Chem. Sci., 2017, 8, 7751-7757.

4 G. Ulrich, A. Barsella, A. Boeglin, S. Niu and R. Ziessel, ChemPhysChem, 2014, 15, 2693-2700.

5 J. Butet, P.-F. Brevet and O. J. Martin, ACS Nano, 2015, 9, 10545-10562.

6 K. Clays and A. Persoons, Phys. Rev. Lett., 1991, 66, 2980.

7 M. Li, Y. Li, H. Zhang, S. Wang, Y. Ao and Z. Cui, J. Mater. Chem. C, 2017, 5, 4111-4122.

8 F. Meyers, S. Marder, B. Pierce and J. Bredas, J. Am. Chem. Soc., 1994, 116, 10703-10714.

9 T.-G. Zhang, Y. Zhao, I. Asselberghs, A. Persoons, K. Clays and M. J. Therien, J. Am. Chem. Soc., 2005, 127, 9710-9720.

10 L. R. Dalton, P. A. Sullivan and D. H. Bale, Chem. Rev., 2009, 110, 25-55.

11 R. D. Fonseca, M. G. Vivas, D. L. Silva, G. Eucat, Y. Bretonnière, C. Andraud, L. De Boni and C. R. Mendonça, J. Phys. Chem. C, 2018, 122, 1770-1778.

12 F. Castet, M. Blanchard-Desce, F. Adamietz, Y. M. Poronik, D. T. Gryko and V. Rodriguez, ChemPhysChem, 2014, 15, 2575-2581.

13 W. Li, Y. Hu, C. Zhong, X. Zhou, Q. Wang, W. Q. Tian and J. D. Goddard, J. Mater. Chem. C, 2016, 4, 6054-6062.

14 S. R. Marder, L.-T. Cheng, B. G. Tiemann, A. C. Friedli, M. Blanchard-Desce, J. W. Perry and J. Skindhøj, Science, 1994, 263, 511-514.
15 R. Andreu, M. J. Blesa, L. Carrasquer, J. Garín, J. Orduna, B. Villacampa, R. Alcalá, J. Casado, M. C. Ruiz Delgado and J. T. López Navarrete, J. Am. Chem. Soc., 2005, 127, 88358845.

16 A. Plaquet, B. Champagne, J. Kulhánek, F. Bureš, E. Bogdan, F. Castet, L. Ducasse and V. Rodriguez, ChemPhysChem, 2011, 12, 3245-3252.

17 O. Ostroverkhova, Handbook of Organic Materials for Electronic and Photonic Devices, Elsevier Science, 2018.

18 B. Gu, C. Zhao, A. Baev, K.-T. Yong, S. Wen and P. N. Prasad, Adv. Opt. Photonics, 2016, 8, 328-369.

19 P. G. Lacroix, I. Malfant and C. Lepetit, Coord. Chem. Rev., 2016, 308, 381-394.

20 F. Mahuteau-Betzer and S. Piguel, Tetrahedron Lett., 2013, 54, 3188-3193.

21 R. Surekha, R. Gunaseelan, P. Sagayaraj and K. Ambujam, CrystEngComm, 2014, 16, 7979-7989.

22 M. Anis, S. Ramteke, M. Shirsat, G. Muley and M. Baig, Opt. Mater., 2017, 72, 590-595.

23 M. Kajamuhideen, K. Sethuraman, K. Ramamurthi and P. Ramasamy, Opt. Laser Technol., 2017, 91, 159-165.

24 C. Bosshard, G. Knöpfle, P. Pretre and P. Günter, J. Appl. Phys., 1992, 71, 1594-1605.

25 D. Burland, C. Walsh, F. Kajzar and C. Sentein, J. Opt. Soc. Am. B, 1991, 8, 2269-2281.

26 P. L. Franzen, L. Misoguti and S. C. Zilio, Appl. Opt., 2008, 47, 1443-1446.

27 L. M. Abegão, R. D. Fonseca, F. A. Santos, G. B. Souza, A. L. B. Barreiros, M. L. Barreiros, M. Alencar, C. R. Mendonça, D. L. Silva and L. De Boni, Chem. Phys. Lett., 2016, 648, 91-96.

28 M. G. Vivas, D. L. Silva, R. D. Rodriguez, S. Canuto, J. r. m. Malinge, E. n. Ishow, C. R. Mendonca and L. De Boni, J. Phys. Chem. C, 2015, 119, 12589-12597.

29 E. Hendrickx, K. Clays and A. Persoons, Acc. Chem. Res., 1998, 31, 675-683.

30 R. Bersohn, Y. H. Pao and H. Frisch, J. Chem. Phys., 1966, 45, 3184-3198.

31 E. Bogdan, A. Plaquet, L. Antonov, V. Rodriguez, L. Ducasse, B. t. Champagne and F. Castet, J. Phys. Chem. C, 2010, 114, 12760-12768.

32 M. de Wergifosse and S. Grimme, J. Chem. Phys., 2018, 149, 024108.

33 W. Kohn and L. J. Sham, Phys. Rev., 1965, 140, A1133.

34 C. Lee, W. Yang and R. G. Parr, Phys. Rev. B: Condens. Matter Mater. Phys., 1988, 37, 785.

35 T. Yanai, D. P. Tew and N. C. Handy, Chem. Phys. Lett., 2004, 393, 51-57.

36 Y. Zhao and D. G. Truhlar, Theor. Chem. Acc., 2008, 120, 215241.

37 J. Tomasi, B. Mennucci and E. Cances, J. Mol. Struct.: THEOCHEM, 1999, 464, 211-226.

38 M. J. Frisch, et al., Gaussian 09 Rev. D01, Gaussian, Inc., Wallingford, 2012.

39 S. Brasselet and J. Zyss, J. Opt. Soc. Am. B, 1998, 15, 257-288. 
40 K. Pielak, F. Bondu, L. Sanguinet, V. Rodriguez, B. Champagne and F. d. r. Castet, J. Phys. Chem. C, 2017, 121, 1851-1860.

41 L. M. Abegão, R. D. Fonseca, T. N. Ramos, F. MahuteauBetzer, S. Piguel, R. José Joatan Jr, C. R. Mendonça, S. Canuto, D. L. Silva and L. De Boni, J. Phys. Chem. C, 2018, 122, 10526-10534.

42 D. L. Silva, R. D. Fonseca, M. G. Vivas, E. Ishow, S. Canuto, C. R. Mendonca and L. De Boni, J. Chem. Phys., 2015, 142, 064312.
43 G. de la Torre, T. Torres and F. Agulló-López, Adv. Mater., 1997, 9, 265-269.

44 X. Liu, Z. Yang, D. Wang and H. Cao, Crystals, 2016, 6, 158. 45 F. A. Santos, L. M. Abegão, R. D. Fonseca, A. M. Alcântara, C. R. Mendonça, M. S. Valle, M. Alencar, K. Kamada, L. De Boni and J. Rodrigues Jr, J. Photochem. Photobiol., A, 2019, 369, 70-76. 